\title{
Wire-localised excision of unpalpable recurrent papillary thyroid carcinoma
}

\author{
Eric Farrell ${ }^{1}$, Richard Speaker ${ }^{1}$, Donal O’Driscoll ${ }^{1}$, and Liam Skinner ${ }^{1}$ \\ ${ }^{1}$ Waterford Regional Hospital
}

May 19, 2020

\begin{abstract}
Ultra sound wire guided markers are safe and effective for unpalpable suspicious nodes Particularly useful on the setting of the previously operated neck Placement by an experienced radiologist in the presence of the operating surgeon is advocated
\end{abstract}

All authors have nothing to declare or disclose.

There are no conflicts of interest to report

3 succinct points:

1. Ultra sound wire guided markers are safe and effective for unpalpable suspicious nodes

2. Particularly useful on the setting of the previously operated neck

3. Placement by an experienced radiologist in the presence of the operating surgeon is advocated

Introduction

The use of wire-localised nodal excision is standard of care in breast cancer patients. The technique has also garnered momentum in other specialties because of its increased accuracy in identifying targets pre operatively, limiting dissection and morbidity associated with its use.

Due to advancing technology and quality of radiological imaging, incidental findings of potentially pathological nodes and lesions are increasing in the field of head and neck surgery. Lesions that are found and reported on scans may not be clinically palpable. Consequentially, subclinical nodes that are identified incidentally or on routine follow up scans create a diagnostic challenge for surgeons and radiologists. In a region as complex as the head and neck where accuracy is a cornerstone of surgical practice, precision is a useful adjunct to mitigate potential morbidity. Further more, in an operative field that has already been exposed to surgery, adjuvant therapy or complications, increased accuracy of nodal biopsy cannot be understated.

Technical note:

This technique was applied in the case of a 38 year old female who had undergone a total thyroidectomy, selective neck dissection and central neck compartment clearance for a pT3N1b multifocal papillary thyroid carcinoma. Her post-operative course was complicated by a chylous leak which was successfully treated with conservative measures and parenteral feeding. Subsequently she underwent radioactive iodine with no focal tracer uptake in the neck or elsewhere after treatment.

Upon routine ultra sound follow up 18 months after primary treatment ended, a unifocal $22 \mathrm{~mm} \times 8 \mathrm{~mm}$ conglomerate lesion was identified. 
It was impalpable on examination. FNA of this mass confirmed nodal recurrence of her papillary thyroid carcinoma. As the node was identified in a previously operated field, ultra sound guided wire localisation (USGWL) was undertaken by a consultant radiologist preoperatively. This is shown in figure 1 below.

The lesion was retrieved through a $3 \mathrm{~cm}$ incision in the previous scar. Following elevation of subplatysmal flaps, the targeted mass was rapidly identified. The stepwise process is outlined in figure 2 below. Histology confirmed 3 nodes of metastatic papillary thyroid carcinoma, the largest of which was $9 \mathrm{~mm}$. The patient did not suffer any complications and was discharged on the same day of surgery. On subsequent follow up she remains well.

\section{Discussion}

The first use of the USGWL technique for head and neck patients was reported by Zimmerman in $2004^{1}$. This technique appears particularly useful in cases that have been previously operated on and in which the operative field may be distorted ${ }^{2}$. Samara et al provided a contemporary summary of the application of USGWL in various pathologies demonstrating the heterogeneity of it's use from oncocytomas to decompressed branchial cysts and thyroglossal duct cysts ${ }^{3}$.

While USGWL has predominantly been reported as a useful pre operative tool, it has also be recorded as a useful intraoperative adjunct in a case of paratracheal nodes that could not be identified during open exploration ${ }^{4}$.

In the management of thyroid nodal recurrence choice of treatment depends on proximity to vital structures, whether the recurrence lies in a previously operated field and the character of the node in question. We advocate a targeted approach to the previously operated on neck. A measured decision regarding suspicious nodes post total thyroidectomy and RAI for papillary thyroid cancer must also be made to retrieve suspicious lymph nodes, particularly in the sub centimetre category as these nodes may have limited potential to progress, with some even regressing as demonstrated in a retrospective review of 166 patients $^{5}$. In our case, the conglomerate of nodes in question was larger than two centimetres and the previously reported histology showed non encapsulated foci of tumour with limited lymphovascular invasion.

The complication rate of USGWL has been minimal in single case reports however in a retrospective review of 17 patients who underwent USGWL for thyroid bed recurrence, complications documented included puncture of the internal jugular vein, vocal cord injury, haematoma, chyle leak and a seroma ${ }^{2}$. In the next largest review, of 8 patients, hookwire localisation was reported to take only 5-10 minutes with all targets being retrieved without complication ${ }^{6}$. Other reported complications include pain and vasovagal response to wire insertion.

Wires should be placed by experienced radiologists, preferably in the presence of the operating surgeon so they can appreciate the location of the target nodes/lesions in question. There is an argument to be made that wires should be placed while the patient is positioned and anaesthetised on the table. This allows (i) anaesthesia for the patient, avoiding pain and vasovagal events (ii) minimise the chance for migration of the wire while patients are themselves moving or being moved in to position on the operating table (iii) increases the chance that both radiologist and surgeon can see exactly the course of the wire and the proximity of nearby structures, this may be particularly pertinent in the previously operated/irradiated neck.

Wire guided localisation has been shown to be an effective adjunct to identifying clinically occult lesions in the head and neck. The application to various pathologies is promising. While the procedure is considered standard of care in other specialties, it has yet to move in to the mainstream in Head and Neck surgery. This is an effective and safe technique in the right hands.

\section{References}

1. Zimmerman P, DaSilva M, Izquierdo R, Cico L, Kort K, Numann P. Intraoperative needle localization during neck reexploration. Am J Surg. 2004;188(1):92-3. 
2. Eng OS, Grant SB, Weissler J, Simon M, Roychowdhury S, Davidov T, et al. Operative bed recurrence of thyroid cancer: utility of a preoperative needle localization technique. Gland Surgery. 2016;5(6):571-5.

3. Samara E, Williams M, Howlett DC. Current applications of ultrasound-guided wire localization in head and neck surgery. Int J Oral Maxillofac Surg. 2019;48(4):443-6.

4. Fletcher AM, Preston TW, Kuehn DM, Clark ED, Van Daele D, Hoffman H. Ultrasound-guided needle localization of recurrent paratracheal thyroid cancer. Ann Otol Rhinol Laryngol. 2009;118(7):475-8.

5. Robenshtok E, Fish S, Bach A, Domínguez JM, Shaha A, Tuttle RM. Suspicious Cervical Lymph Nodes Detected after Thyroidectomy for Papillary Thyroid Cancer Usually Remain Stable Over Years in Properly Selected Patients. The Journal of Clinical Endocrinology \& Metabolism. 2012;97(8):2706-13.

6. Park JY, Park NH, Yi SY, Ko MS, Park HJ. Preoperative US-guided hookwire localization for nonpalpable cervical masses. J Clin Ultrasound. 2012;40(4):195-9.

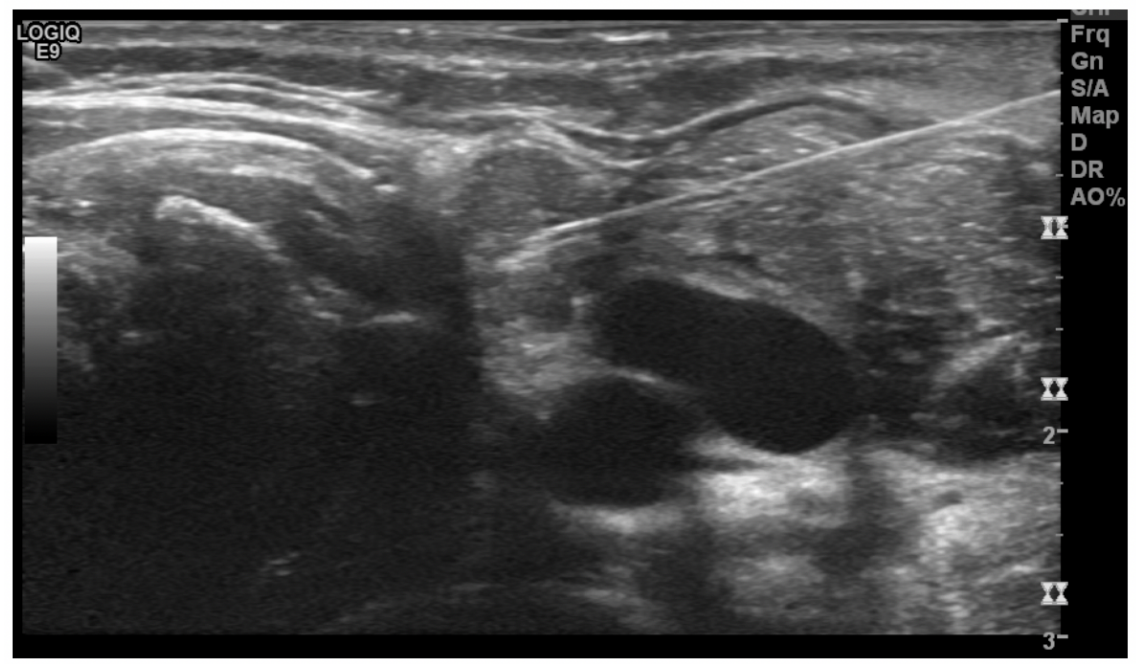

Figure 1 Node being marked with a needle 


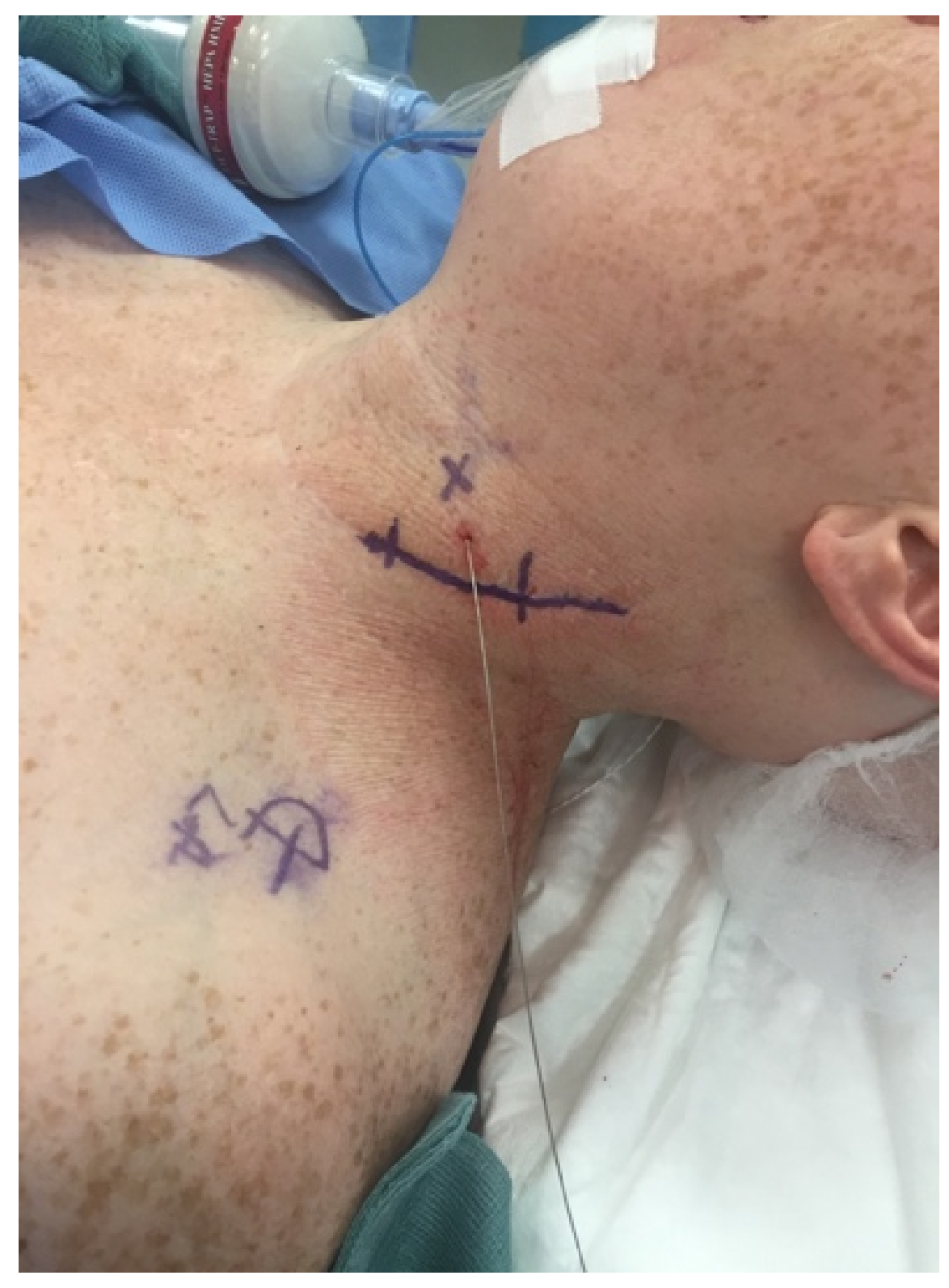

